\title{
BMJ Open Shanghai Preconception Cohort (SPCC) for the association of periconceptional parental key nutritional factors with health outcomes of children with congenital heart disease: a cohort profile
}

\author{
Dingmei Wang, ${ }^{1}$ Yi Zhang, ${ }^{1,2}$ Yuang Jiang, ${ }^{1}$ Ying Ye, ${ }^{1} \mathrm{Mi} \mathrm{Ji},{ }^{1}$ Yalan Dou, ${ }^{1}$ \\ Xiaotian Chen, ${ }^{1}$ Mengru Li, ${ }^{1}$ Xiaojing Ma, ${ }^{1,2}$ Wei Sheng, ${ }^{1,2}$ Guoying Huang, ${ }^{1,2}$ \\ Weili Yan (1) , 1,2 On behalf of the SPCC group
}

To cite: Wang D, Zhang $Y$, Jiang $Y$, et al. Shanghai Preconception Cohort (SPCC) for the association of periconceptional parental key nutritional factors with health outcomes of children with congenital heart disease: a cohort profile. BMJ Open 2019;9:e031076. doi:10.1136/ bmjopen-2019-031076

- Prepublication history and additional material for this paper are available online. To view these files, please visit the journal online (http://dx.doi org/10.1136/bmjopen-2019031076).

DW and YZ contributed equally.

Received 15 April 2019

Revised 13 October 2019

Accepted 24 October 2019

Check for updates

(c) Author(s) (or their employer(s)) 2019. Re-use permitted under CC BY-NC. No commercial re-use. See rights and permissions. Published by BMJ.

${ }^{1}$ Children's Hospital of Fudan University, Shanghai, China

${ }^{2}$ Shanghai Key Laboratory of Birth Defect, Shanghai, China

Correspondence to Dr Weili Yan;

yanwl@fudan.edu.cn

Dr Guoying Huang; gyhuang@shmu.edu.cn

\section{ABSTRACT}

Purpose The Shanghai Preconception Cohort (SPCC) was initially established to investigate the associations of parental periconceptional nutritional factors with congenital heart disease (CHD) but has further analysed child growth and development and paediatric diseases. Participants Preparing-for-pregnancy couples who presented at Shanghai preconception examination clinics and early-pregnancy women before 14 gestational weeks were enrolled to comprise the periconceptional baseline study population. General characteristics, routine clinical data and consumption of diet supplements, such as folic acid and multivitamins, were collected. Blood samples were obtained at preconception and early, middle and late gestations using standard procedures. Multiple nutritional factors, including folate, homocysteine, vitamin $A$, vitamin $D$, vitamin $E$ and metals, in the blood samples of participants selected using a case-control design were examined. Genomic DNA was extracted.

Findings to date The baseline population included 8045 preconception couples, 3054 single women and 15615 early-pregnancy women. Data from 12402 births were collected, and follow-up of the cohort for other outcomes is ongoing. Currently, 151 cases of $\mathrm{CHD}$ were identified after birth. The pilot analysis in a small subgroup showed that approximately $20.0 \%$ of preconception women and $44.9 \%$ of early-pregnancy women had red blood cell (RBC) folate levels that met the international recommendation for preventing neural tube defects.

Future plans Once a sufficient number of $\mathrm{CHD}$ cases are achieved, we will investigate the quantitative association of preconception RBC folate levels with $\mathrm{CHD}$ using a nested case-control design. The SPCC will be followed up for 18 years to investigate extensive outcomes of growth, development, obesity, and common and rare diseases during childhood and adolescence according to our plan. Blood nutritional factors will be examined in participants selected for specific aims. The SPCC will also allow for prospective cohort studies on extensive research questions.

Trial registration number NCT 02737644
Strengths and limitations of this study

- The Shanghai Preconception Cohort is the first prospective birth cohort with congenital heart disease as primary outcome with recruitment starting from the preconception stage. Temporal sequence of exposures and outcomes can be achieved for causal inference of birth defects and other diseases that develop during the early stage of gestation.

- Preconception blood samples were appropriately collected and stored, which allow for the examination of individual blood levels for nutritional factors and other exposures.

- Preconception clinical data and blood samples from both the father and mother were collected to determine the effect of both maternal and paternal genetic and nutritional factors on fetal and paediatric diseases.

- Although the response rate was high (>95\%), preconception participants were recruited from the population who voluntarily presented at preconception physical examination sites in Shanghai. They may have stronger willingness for a healthy pregnancy, which may induce selection bias.

- Biological samples (cord blood and placenta) of the newborns were not collected.

\section{INTRODUCTION}

Congenital heart disease (CHD) is a common congenital malformation that seriously affects children's quality of life. ${ }^{1}$ CHD is a leading cause of infant death in high-income countries, affecting 8 of 1000 live births. ${ }^{2}$ According to the report from National Health and Family Planning Commission of the People's Republic of China, CHD accounts for about a quarter of the birth defects of newborns in China, ranking first among birth defects. ${ }^{3}$ In a prospective, nationwide large-scale study in >120 000 newborns in China in 2013, the 
prevalence of CHD in live births was $8.94 \%$. The incidence of severe CHD was $2.9 \% .^{4}$

The cause of CHD is multifactorial. With the development of genetic engineering technology, genetic factors have been better understood in the past decade. ${ }^{5}$ Multiple factors have been reported in epidemiological studies, including maternal social variables (occupation and educational background), health status, unhealthy lifestyle, maternal medical history, emotional status, family history of the disease, and consanguineous marriages. ${ }^{6-10}$ Additionally, maternal key nutrients are related to the risk of offspring's CHD as a modifiable environmental factor during periconception. ${ }^{11}{ }^{12}$ The periconceptional intake of folic acid supplements has been shown to reduce the risk of CHD, ${ }^{1314}$ and the use folic acid supplements before conception and at the beginning of pregnancy has been recommended to women worldwide. Awareness of the relationship between folic acid deficiency and CHD is actually a by-product finding from the well-known Hungarian randomised control trial study of folic acid supplementation to prevent neural tube defects. The study found that prenatal supplementation with a multivitamin containing $0.8 \mathrm{mg}$ of folic acid daily reduced the incidence of neural tube defects. Additionally, the incidence of various heart defects has also been reduced by nearly half. ${ }^{15}$ Longitudinal data from $>1000000$ births in Canada in 22 years, from 1990 to 2011, also showed that food fortification with folic acid reduced the risk of CHD by $20 \%-30 \%{ }^{14}$ The current guideline for folic acid supplementation recommends that all women of childbearing potential be supplemented with at least $0.4 \mathrm{mg}$ of folic acid daily prior to conception and during pregnancy, which is designed to prevent neural tube defects. ${ }^{16}$ However, excessive folic acid intake may increase the risk of cancer, ${ }^{17}$ vitamin $\mathrm{B}_{12}$ deficiency ${ }^{18}$ and autism spectrum disorder. ${ }^{19}$ The optimal dose of folic acid for preventing CHD warrants further investigation. Additionally, most previous studies focused only on the folic acid supplement or the serum folate level during or after pregnancy, which may not be the optimal period and method to reflect the exposure level to the risk of CHD.

To investigate the association between parental periconceptional key nutritional factors, such as folate level, and the development of CHD and to explore the cut-off biomarker levels, we conducted the Shanghai Preconception Cohort (SPCC) and a nested case-control analysis.

The SPCC was initiated primarily to evaluate CHD. However, based on the strengths of its baseline data collection, it has received attention and support, with improved additional extensive outcomes for children that will be followed up for a longer term.

\section{COHORT DESCRIPTION}

\section{Who is in the cohort?}

The SPCC recruited pregnancy-planning women and men who were permanent residents and voluntarily presented at preconception clinical clinics at 28 maternity institutions in 10 districts of Shanghai (Minhang District, Huangpu District, Xuhui District, Changning District, Jing'an District, Putuo District, Yangpu District, Pudong District, Songjiang District and Qingpu District) from March 2016 to December 2018. The preconception examination policy in the city of Shanghai provides a unique opportunity and clinical resources to support recruitment in the SPCC. Since 2010, married couples in Shanghai have been encouraged to attend a free preconception health examination. Moreover, these maternity institutions receive strong local administrative support and integrated maternal healthcare networking, providing service to 150 000-200 000 annual deliveries in Shanghai. Couples who were present at preconception clinics, living in Shanghai, preparing for pregnancy within 1 year, and planning to receive antenatal care and deliver in Shanghai were eligible for the study. Written informed consent was obtained from all participants before data collection. Additionally, we recruited early-pregnancy women who were at $<14$ gestational weeks at their first antenatal examination. These two groups of participants comprised the periconceptional baseline study population.

The first primary outcome of the SPCC is CHD. The hypothesis is that maternal preconception serum or red blood cell (RBC) folate level is quantitatively associated with CHD development in the offspring. The study design and protocol have been registered in the ClinicalTrials Registry.

As shown in figure 1, the baseline population will be followed up until delivery, and their infants will be followed up until 18 years of age (figure 1).

\section{Follow-up procedure}

On enrolment, the participants completed the questionnaire of key nutrient supplementation and blood sample collection. When participants became pregnant, the same investigations (questionnaire/blood sample collection) were conducted during early pregnancy (first antenatal visit at 16-20 gestational weeks). Pregnancies were followed up, along with routine maternal healthcare procedures. Blood samples were also collected at the second (24-28 gestational weeks) and third (32-34 gestational weeks) trimesters. The follow-up data of CHD outcome and birth were obtained through the Shanghai Neonatal CHD Screening Platform (figure 1).

As shown in figure 1, outcomes at birth, from infancy to childhood (preschool phase) and between 7 and 18 years (school ages), will be collected or extracted from multiple public platforms and data sources. First, preconception clinical visit data from the Preconception Care Electronic Data System, supported by the national and local government, were collected, including height, weight, age, infections, sexually transmitted disease and family history. Second, the routine pregnancy data were obtained from the Maternal Clinic Antenatal Medical Record System, managed by the Shanghai Center for Women and Children's Health, including height, gestational weight, last menstrual period, childbearing history, delivery outcomes, infections, haematocrit, coagulation 


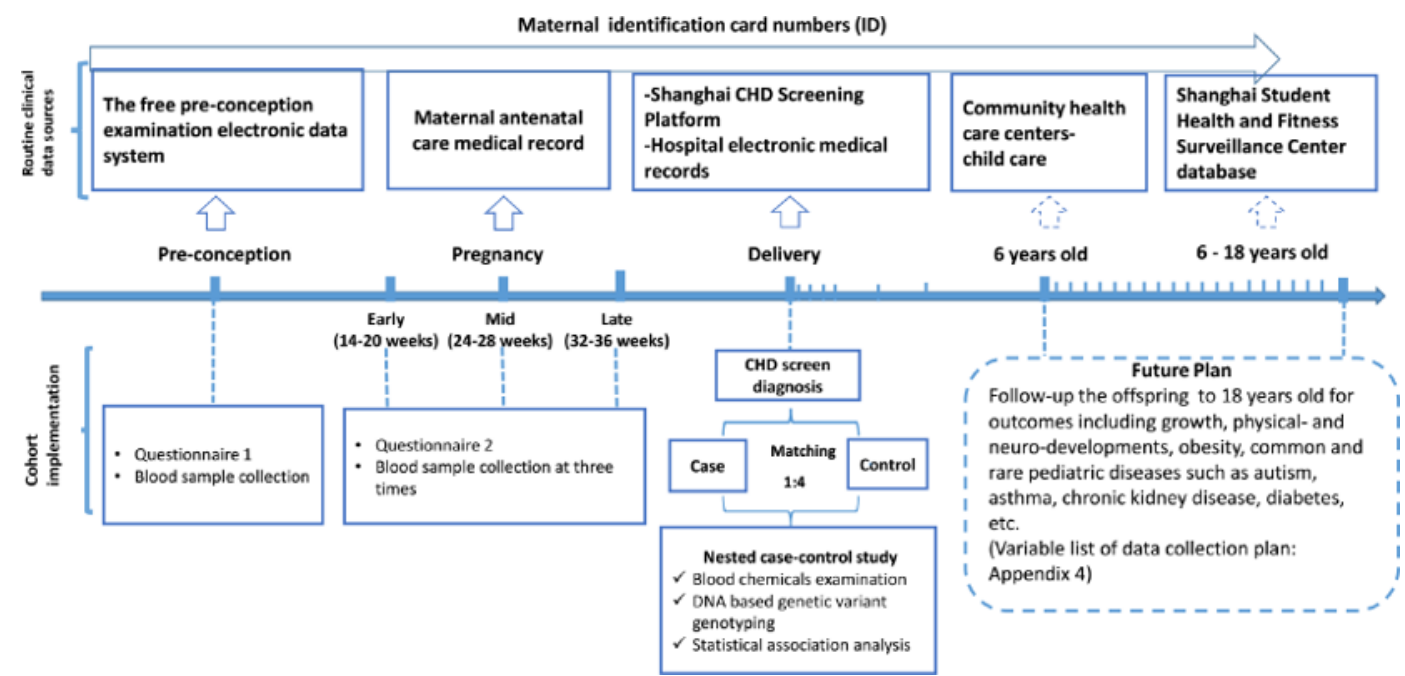

Figure 1 Protocol and follow-up of the SPCC. The baseline population of SPCC consisted of women and men at the periconception stage (couples who are preparing for pregnancy) and pregnant women at the early gestation stage. The cohort includes three phases, from periconception to birth (phase I, perinatal phase), from birth to 6 years (phase II, infant and preschool age) and from 7 to 18 years (phase III, school age). The current paper focuses on the first phase, with CHD as the primary outcome, and will cover other folate-sensitive birth defects. CHD, congenital heart disease; SPCC, Shanghai Preconceptional Cohort.

function, and liver and kidney functions. Lastly, the maternal and neonatal data at delivery were obtained from the Shanghai Neonatal CHD Screening Platform, including birth weight, CHD diagnosis, birth defects and Apgar score. Additionally, we will work with the Shanghai Student Health and Fitness Surveillance Center to obtain outcome data. The personal national identification card numbers of the participants are applied as index variables through multiple data sources. The detailed variable list and codebook of data collection are presented in online supplementary appendix 1 .

During the first phase of the cohort, from preconception to delivery, comprehensive strategies were used to retain participants in the study. For mothers, we provided a variety of engagement activities, including green channel (fast track) to their antenatal care, to provide convenience and to save their time in hospitals. We also provided a contact number on the participant card to answer their calls or queries about the study procedures. Site investigators at early-pregnancy clinics in collaborative hospitals were provided a smartphone application to help timely identification of recruited cohort participants and to manage data and blood sample collection procedures. We also provided green channel echocardiography for diagnosing CHD in all site hospitals to enhance the compliance of participants. Moreover, an automated text message system was adopted to remind participants of schedules and follow-up appointments.

\section{Study measures}

Personal characteristics questionnaires

As shown in figure 1 , questionnaire 1 was administered during recruitment at preconception examination sites, and questionnaire 2 was administered at early-pregnancy sites to collect information on the consumption of folic acid and vitamin supplements, and on the brand and content of nutritional supplement. Information on the demographics, maternal education, sociodemographic status, occupation, smoking status, alcohol consumption, body mass index, medication and health status was also included. In addition to the content of questionnaire 1, questionnaire 2 added drug information, reproductive history and health status. Questionnaire 1 for baseline and questionnaire 2 for the first antenatal visit at early pregnancy are presented in online supplementary appendix $2 \mathrm{~A}, \mathrm{~B}$.

\section{Blood sample collection}

In this study, the remaining blood samples for routine clinical blood examination were collected. The blood sample for routine clinical examination was usually $5 \mathrm{~mL}$ and was extracted in the morning. Routine clinical blood examination was performed at room temperature $\left(20^{\circ} \mathrm{C}-\right.$ $25^{\circ} \mathrm{C}$ ). The remaining samples (fasting serum and EDTA anticoagulation) of peripheral venous blood from routine laboratory clinical blood examination were retained. These blood samples were temporarily stored in a refrigerator at $4^{\circ} \mathrm{C}$ for dispensing within 6 hours. After completion of blood sample distribution, the serum and whole blood were stored at the site laboratory in a refrigerator at $-20^{\circ} \mathrm{C}$ and then transported by three trained investigators to the central laboratory for storage in freezers at $-80^{\circ} \mathrm{C}$ for 2 weeks. Sampling tubes were made of a light-proof material, and the process of collecting blood samples was completely protected from light.

\section{Examination of key nutritional factors in blood samples}

The examinations will be conducted in participants selected by nested case-control designs based on specific aims. 
$R B C$ folate, serum folate, serum homocysteine, vitamin $D$, vitamin $B_{12}$ and serum ferritin

EDTA anticoagulated blood samples were collected to measure RBC folate, serum folate, serum homocysteine, vitamin $D$, vitamin $B_{12}$ and serum ferritin. All six biomarkers were analysed using electrochemiluminescence assays (ARCHITECT i2000SR Analyzer; Abbott Laboratories, USA). A standard solution with known level (produced by Abbott Laboratories) was used daily to control the quality before the measurement. If the quality control level was out of range, the measurement would be suspended and adjusted. External quality control was conducted with the control laboratory data programme from Abbott Laboratories (Abbott Laboratories, Shanghai, China). RBC folate levels were adjusted for haematocrit. If the RBC folate level is $<126.0$ or $>651.1 \mathrm{ng} / \mathrm{mL}$, adjustment was needed based on the serum folate level. The haematocrit data were extracted from the hospital laboratory information system. These examinations were performed in the central laboratory of the Children's Hospital of Fudan University.

\section{Vitamins $A$ and $E$}

The serum vitamin A and $\mathrm{E}$ levels were quantitatively determined by liquid chromatography-tandem mass spectrometry (LC-MS /MS) in the central laboratory of the Children's Hospital of Fudan University. The testing instrument was triple quadrupole mass spectrometer LC-MS/MS system (API 3200MDTM, AB Sciex Pte). A standard solution of vitamin $\mathrm{A}-\mathrm{d} 6$ and $\mathrm{E}-\mathrm{d} 6$ was applied as an internal standard.

\section{Glycaemic and lipid profiles}

Fasting serum cholesterol, high-density lipoprotein, lowdensity lipoprotein, triglyceride and glucose levels were measured using the Beckman Coulter AU chemistry analysers (Beckman Coulter, USA) in the central laboratory of the Children's Hospital of Fudan University.

\section{Metals}

Serum levels of Mg, Fe, Zn, SE, Mn, As, Cu and Ca were analysed by inductively coupled plasma mass spectrometry (Inductively Coupled Plasma Optical iCAP6300; Thermo Scientific, USA) in standard mode..$^{20}$ The metal examination was conducted in the Instrumental Analysis Centre of Shanghai Jiao Tong University, which is a national key laboratory.

\section{Genomic DNA extraction}

Genomic DNA of all participants was extracted using a magnetic bead-based kit (TGuide M16 Automatic Nucleic Acid Extractor (OSE-M16); Tiangen Biotech (Beijing) Co., China) from $2 \mathrm{~mL}$ of EDTA anticoagulated whole blood sample after routine blood examination. Genomic DNA samples were stored for future studies. An average of 150 ng DNA was available. Similar to that of blood chemicals, future genetic variant genotyping will be performed in selected participants according to the nested case-control design for specific aims. Currently, there are no candidate genes or variants that are listed.

\section{Outcomes: CHD in neonates}

The diagnosis of CHD was the primary outcome of the study at this stage and was obtained from the Shanghai Neonatal CHD Screening Platform, which was initiated as a routine screening tool for newborns in Shanghai since 1 June 2016. The standard protocol of CHD screening in the platform was previously described in detail. ${ }^{21}$ All newborns underwent the screening using a double-index method (ie, cardiac murmur auscultation and pulse oximetry) at 6-72 hours after delivery, and screening-positive newborns would undergo subsequent echocardiography for further confirmative diagnosis.

SPCC will also collect data on other birth defects as secondary outcomes, including Down's syndrome, neural tube defects, hydrocephalus, digestive tract malformations, urinary malformations and behavioural-cognitive developmental disorder. After delivery, the infants underwent routine childcare procedures organised by the Shanghai Child Healthcare System, which are administered by the Shanghai Center for Women and Children's Health. All records of birth defects, which were diagnosed after birth, as well as routine neurodevelopmental examinations and longitudinal anthropometric data, were abstracted from the system by a professional clinical team from the Children's Hospital of Fudan University (for details of the types of birth defects, please see online supplementary appendix 3)

\section{Statistical methods}

To investigate the association of maternal preconception nutrition factor levels with offspring CHD risk, a nested case-control study was conducted. The control was matched by age and site.

The sample size for the nested case-control analysis was planned as $180 \mathrm{CHD}$ cases and 720 matched controls to detect maternal folate deficiency with a prevalence of $50 \%$ in controls (with an OR of 1.6) to achieve a power of $80 \%$ at an alpha of 0.05 . Based on a CHD incidence $>8.94$ per 1000 live births, ${ }^{4} 20000$ pregnancies were needed. For a continuous nutrient variable with SD of 2.0, 50 matched pairs (1:4) were required to achieve $90 \%$ power to detect an OR of 1.3, calculated using conditional logistic regression with a 0.05 significance level. ${ }^{22} 23$ Once a sufficient number of CHD cases were achieved, the quantitative association of preconception RBC folate levels with CHD development using nested case-control design was investigated.

Conditional multivariate logistic regression was used in the association analysis with offspring CHD status being the dependent variable and nutrition factor levels as exposure variables, after adjustment for all potential paternal and maternal covariates. ORs and 95\% CIs were reported. To explore a potential cut-off point of the nutrition factor levels that significantly increases the risk of CHD, a dummy variable was set by categorising the maternal preconception nutrition factor levels based on the distribution of the control group. The dose-response relationship was also analysed. Sensitivity analysis included non-conditional 
logistic regression analysis or generalised estimating equation models or generalised linear models, when necessary.

\section{FINDINGS TO DATE}

The SPCC started recruitment in March 2016. As shown in figure 2, by December 2018, we consecutively recruited 19 144/19 $563(97.9 \%)$ participants at preconception settings, including 8045 couples and 3054 single women, and an additional 15 615/16 201 (96.4\%) pregnant women at maternity hospitals with a gestational age $\mathrm{f} o<14$ weeks. Table 1 describes the basic demographic characteristics of the preparing-for-pregnancy participants and pregnant women. The average age of the preconception population was 29.9 (SD 3.9) years for women and 31.4 (SD 4.5) years for men; $31.4 \%$ of men and $2.2 \%$ of women were smokers; and
$61.4 \%$ of men and 30.9 of women had an alcohol drinking habit. In pregnant women, the average age was 29.9 (SD 4.0) years, with half having their first pregnancy. Preconception women were similar in age but different in education levels and occupation. The prevalence of smoking and alcohol drinking was much lower. The descriptive data of table 1 are partly included in another manuscript.

By the end of December 2018, the last participants recruited at early pregnancywere due for delivery; however, we have obtained birth records of only 12402 newborns. The follow-up of outcomes of the remaining participants are ongoing (shown in figure 2). A total of 151 cases of CHD were identified through the CHD screening platform: 131 cases from the early-pregnancy sample and the remaining 20 cases from the preconception sample. The

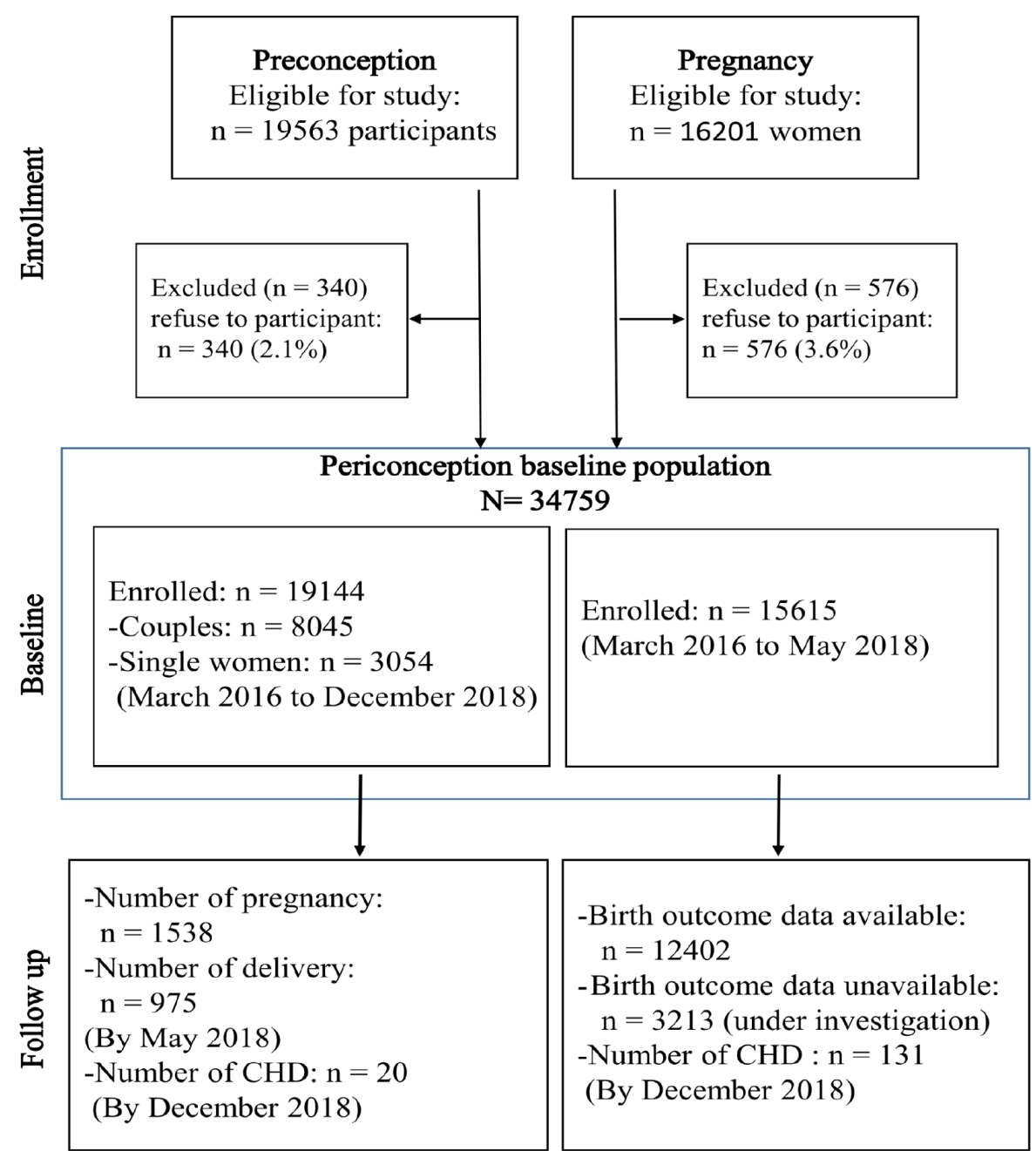

Figure 2 Flowchart showing that by December 2018, we consecutively recruited 19 144/19 563 (97.9\%) participants at preconception settings, including 8045 couples and 3054 single women at prepregnancy sites, and additional $15615 / 16$ 201 (96.4\%) pregnant women at maternity hospitals with a gestational age of $<14$ weeks were recruited. By the end of December 2018, the last participants recruited at early pregnancy were due for delivery. We have achieved birth records of 12402 newborns in the Maternal Clinic Antenatal Medical Record System. The follow-up of outcomes of the remaining participants is ongoing. A total of 151 cases of CHD were identified through the Shanghai Neonatal CHD Screening Platform: 131 cases from the early-pregnancy sample and the remaining 20 cases from the preconception sample. The prevalence of CHD in live births is $10.5 \%$ o (131/12 402) based on the present available data. In the preconception sample, from March 2016 to May 2018 (the latest data extraction in the Maternal Clinic Antenatal Medical Record System), the numbers of pregnancies and deliveries were 1538 and 975 , respectively. CHD, congenital heart disease. 

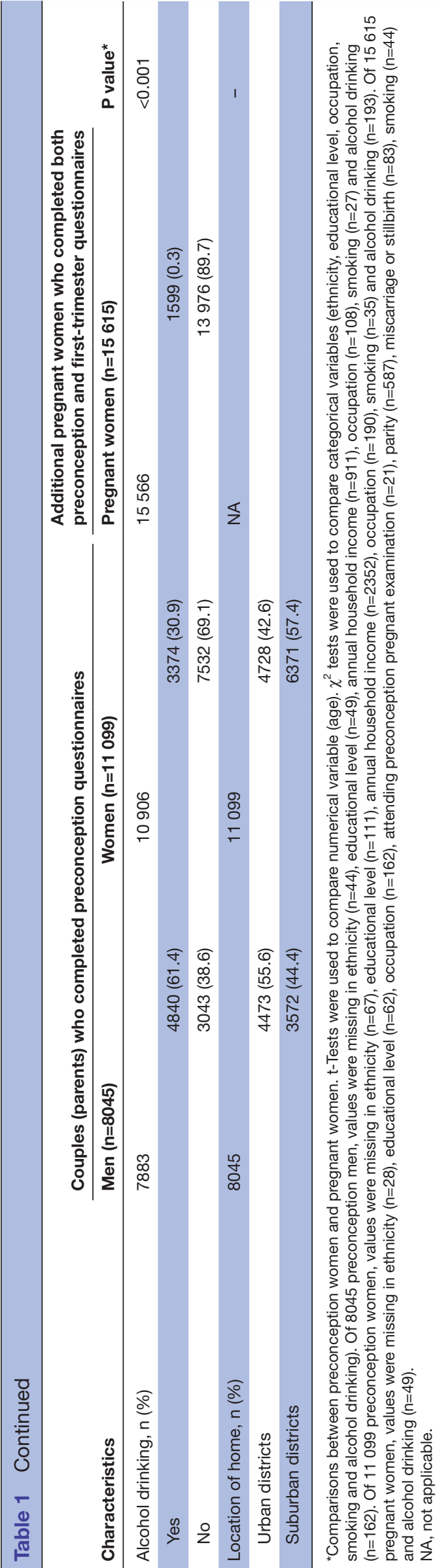

prevalence of CHD in live births is $10.5 \%$ o (131/12402) based on the present available data.

We conducted a small pilot study in April 2017 to determine blood levels of nutritional factors, including serum folate, $\mathrm{RBC}$ folate, vitamin $\mathrm{A}$, vitamin $\mathrm{E}$ and vitamin $\mathrm{D}$. The blood samples from 627 women were selected consecutively from the preconception sample according to those who were identified as pregnant. Additionally, 597 women who were consecutively recruited from the antenatal care clinics were selected. As shown in table 2, the median RBC folate levels were $247.0 \mathrm{ng} / \mathrm{mL}$ (IQR $184.8-340.5 \mathrm{ng} / \mathrm{mL}$ ) in preconception women and $417.4 \mathrm{ng} / \mathrm{mL}$ (IQR 308.6$544.2 \mathrm{ng} / \mathrm{mL}$ ) in early-pregnancy women. Moreover, $20.0 \%$ of preconceptional participants and $44.9 \%$ of pregnant participants had a folate level of $>400 \mathrm{ng} / \mathrm{mL}$, which was suggested as the optimal level for preventing neural tube development defects. ${ }^{24}{ }^{25}$ These results suggest that effort is urgently needed to improve folic acid supplementation in the preparing-for-pregnancy population, especially before pregnancy.

Based on the SPCC, the possible scope of research questions and available types and number of biosamples and biomarkers that can be examined are shown in table 3 .

\section{FUTURE PLANS}

We have a complete plan to follow up offspring until the age of 18 years. The cohort will be financially supported by different grants. The current manuscript focuses on the first phase, the establishment of the baseline and our first main outcome, CHD. The data collection plan for infants and children (from birth to 6 years (preschool stage) and from 6 to 18 years (school age)) is included. During the stage of $0-6$ years, the neurodevelopmental data will be collected from routine childcare clinical visits at birth; at 6 weeks; and at $6,12,36$ and 60 months through the cooperating medical institutions. Physical measurement data and dietary intake information can also be collected at this stage. During the stage of 6-18 years, we plan to follow up on their growth (height, weight and blood pressure), mainly relying on the annual physical examination results of the Shanghai Student Health and Fitness Surveillance Center System. Multiple outcomes for children, including growth and development, cardiovascular diseases, neurodevelopment, metabolic diseases, obesity and hypertension, will be investigated. Please see online supplementary appendix 4 for details.

\section{STRENGTHS AND LIMITATIONS}

Compared with existing birth cohorts, there are three important strengths in our cohort. First, the SPCC is the first prospective birth cohort with CHD as primary outcome and recruitment starting from preconception. Blood samples were collected and stored, which allows direct measurement of individual exposure levels before the development of CHD and causal inference. Temporal sequence of exposures and outcomes can be achieved for causal inference 
Table 2 Distributions of biomarkers in women before and at early gestation (pilot study)

\begin{tabular}{|c|c|c|c|c|}
\hline \multirow[b]{2}{*}{ Biomarker } & \multicolumn{2}{|c|}{ Preconception } & \multicolumn{2}{|c|}{ Early pregnancy } \\
\hline & $n$ & Level & $\mathbf{n}$ & Level \\
\hline Serum folate $(\mathrm{ng} / \mathrm{mL})$, median (IQR) & 620 & $9.7(6.5-13.8)$ & 577 & $14.5(11.2-16.4)$ \\
\hline $\begin{array}{l}\text { Red blood cell folate }(\mathrm{ng} / \mathrm{mL}) \text {, median } \\
\text { (IQR) }\end{array}$ & 570 & $247.0(184.8-340.5)$ & 587 & $417.4(308.6-544.2)$ \\
\hline Homocysteine ( $\mu \mathrm{mol} / \mathrm{L})$, median (IQR) & 624 & $6.5(5.2-8.6)$ & 599 & $4.2(3.5-5.2)$ \\
\hline Vitamin $B_{12}(p g / m L)$, median (IQR) & 625 & $495.2(394.2-639.0)$ & 600 & $388.5(289.4-511.4)$ \\
\hline Vitamin D ( $\mathrm{ng} / \mathrm{mL})$, mean $\pm S D$ & 607 & $16.3 \pm 6.0$ & 578 & $15.5 \pm 6.1$ \\
\hline
\end{tabular}

of birth defects and other diseases that develop during the early stage of gestation. To date, no published studies have measured maternal blood folate levels before conception and associated it with disease outcomes. Second, this cohort also allows the investigation of associations between periconceptional maternal and paternal nutrition exposures and other birth defects, early-onset diseases and neurodevelopmental outcomes. Preconception blood samples were appropriately collected and stored, which allows the examination of individual blood levels of nutritional factors and other exposures. Lastly, both paternal and maternal clinical data and blood samples before conception were collected, which will allow for the testing of the effects of both maternal and paternal genetic and nutritional factors on fetal and paediatric diseases.

Three limitations of this cohort study should be considered. First, there are approximately 200000 pregnant women giving birth annually in Shanghai, and approximately 20000 of them will participate in the free preconception care in Shanghai, where participants were recruited consecutively. Although the response rate was high $(>95 \%)$, preconception participants were recruited from a population who voluntarily presented in Shanghai preconception physical examination sites, who may have a stronger willingness for a healthy pregnancy. This may induce selection bias. Second, in this study, biological samples (cord blood and placenta) of the newborns are not collected. We plan to obtain new informed consent to the family who are willing to participate in future studies to collect biological samples not previously mentioned. Furthermore, electrochemiluminescence assay was used to examine serum and RBC folate levels, which are different from the widely used microbiological assay. This will not result in bias in the association analysis, but comparison with international populations needs caution.

Table 3 Biosamples collected and biomarkers that can be examined in the Shanghai Preconceptional Cohort

\begin{tabular}{|c|c|c|c|c|}
\hline \multirow[b]{2}{*}{$\begin{array}{l}\text { Biosamples available in } \\
\text { participants }\end{array}$} & \multirow[b]{2}{*}{$\begin{array}{l}\text { Available sample type and } \\
\text { volume }\end{array}$} & \multicolumn{3}{|l|}{ Time } \\
\hline & & $\begin{array}{l}\text { Preconception+early } \\
\text { pregnancy (baseline) }\end{array}$ & 24-28 weeks & 32-36 weeks \\
\hline \multirow[t]{4}{*}{ Mother } & & $(n=25487)$ & $(n=8668)$ & $(n=7522)$ \\
\hline & Serum, $600 \mu \mathrm{L}$ & Yes & Yes & Yes \\
\hline & Whole blood, $1 \mathrm{~mL}$ & Yes & Yes & Yes \\
\hline & Genomic DNA, $150 \mathrm{ng}$ & Yes & Yes & Yes \\
\hline \multirow[t]{4}{*}{ Father } & & $(n=7151)$ & - & - \\
\hline & Serum, $600 \mu \mathrm{L}$ & Yes & NA & NA \\
\hline & Whole blood, $1 \mathrm{~mL}$ & Yes & NA & NA \\
\hline & Genomic DNA, $150 \mathrm{ng}$ & Yes & NA & NA \\
\hline Child & NA & & & \\
\hline
\end{tabular}

Scope of research questions: (1) quantitative association of preconception key nutrition factor levels (eg, serum and RBC folates and related biomarkers, such as homocysteine and vitamin $\mathrm{B}_{12}$ ) with congenital heart disease, currently, and other folate-sensitive birth defects; (2) quantitative association of periconceptional maternal and paternal key nutrition factor levels (preconception and dynamic levels during gestation) with important maternal and neonatal gestational complications, neurodevelopment of infants, childhood obesity and clinical paediatric diseases; (3) periconceptional maternal and paternal folate levels with autism spectrum disorder, allergy and asthma in children. Biomarkers that will be examined in different types of biosamples: (1) biomarkers based on serum sample: (a) folate and related markers: serum folate and homocysteine; (b) other vitamins: vitamin A, vitamin $B_{12}$, vitamin $E$ and vitamin $D$; (c) macrometals and micrometals: Mg, Fe, $\mathrm{Zn}, \mathrm{Se}, \mathrm{Mn}, \mathrm{As}, \mathrm{Cu}$ and $\mathrm{Ca}$ (in mg/L); (d) serum ferritin; (e) fasting glycaemic and lipid profiles: fasting glucose, total cholesterol, triglyceride, high-density lipoprotein and low-density lipoprotein; (2) whole blood sample: RBC folate; and (3) genomic DNA sample: candidate genetic variants or genome-wide variants are possibly examined.

$\mathrm{NA}$, not applicable; RBC, red blood cell. 


\section{COLLABORATION}

Investigators with an interest in hypotheses related to SPCC (who meet the requirements of current approvals) are welcome to contact Dr Guoying Huang or Weili Yan. A 'research collaboration application' should be sent to the corresponding author by email. The application should include a brief description of the project.

Acknowledgements We are extremely grateful to all participants in this study, physicians for their help in the recruitment and the whole Shanghai Preconceptional Cohort team, which includes interviewers, computer and laboratory technicians, clerical workers, research scientists, volunteers, managers, receptionists and nurses. We thank Editage (www.editage.com) for English language editing.

Collaborators SPCC group: Guoying Huang, Weili Yan, Xiaojing Ma, Weifen Luo, Wei Sheng, Yi Zhang, Yuan Jiang, Yin Ye, Dingmei Wang, Xiaotian Chen, Mengru Li, Mi Ji, Yumei Liu, Gu Qing(s), Gu Qing(o), Linmei Zhu, De'ai Hou, Peiyu Sun. (Children's Hospital of Fudan University, Shanghai, China), Hongbing Wang, Li Meng, Lin Zhang (Jingan Maternal and Child Health Center), Zifen Dai, Li fen (Shanghai First Maternity and Infant health Hospital), Shufang Chen, Zhenhua Tang, Jiahao Wu (International Peace Maternal and Child Health Hospital), Shuhua Wang, Dan Ii, Hui Wang (Xuhui Maternal and Child Health Center), Yu Ke, Weiping Cao, Baoren Zhang, Hong Huang (Shanghai Pudong New Area Health Care Hospital for Women \& Children), Nailing Wang, Min Jiang, Jie Chen, Qiumin Xia (Shanghai Punan Hospital of Pudong New District), Hui Xu, Guoying Lao (Changning Maternity and Infant Health Hospital), HongMei Jin, Wenjuan Xie, Pin Yi (Qingpu Hospital, Zhongshan Hospital), Weiming Gong, JianXin Xu, Yingying Qian (Shanghai Qingpu Maternal and Child Health Center), Mingjie Luo, Jingwei Xia, Dongmei Chen, Zhenyu Tang (Shanghai Huangpu Maternal and Child Health Center), Xuejing Zhu, Qing Liu, Huiling Yang (Shanghai Huangpu Maternal and Child Health Hospital), Xiaotian Li, Zhiyong Wu, Chuanmin Ying, Shan Shi (Obstetrics and Gynecology Hospital of Fudan University (Shanghai Red House Obstetrics and Gynecology Hospital)), Yanquan Zhang, Mingyi Yang (Wujing Hospital, Minhang District, Shanghai), Xiaohua Zhang, Lei Zhang, Lin Guan (Shanghai Minhang District Maternal and Child Health Care Hospital), Jinyu Xu, Honglin Wang, Fang Shen (The Fifth People's Hospital of Shanghai, Fudan University), Wenying Li, Xiaojing Teng, Jinling Zhao (Shanghai Minhang TCM Hospital), Cuili Zhu, Lan Wang, Hongwei Chen (Shanghai Songjiang District Central Hospital), Xiaoming Yuan, Meihua Zhang, Yaqiong Jin (Sijing Hospital, Songjiang District, Shanghai), Qing Yang, Hong Zhu, Min Feng (Songjiang Maternal and Child Health Center), Ying Wang, Yan Wu, Hong Tang (Songjiang Maternal and Child Health Hospital), Sa Guo (Tongji Hospital of Tongji University), Hongling Du (Shanghai Putuo District People's Hospital), Yuhuan Liu, Zhanyue Yi, Renhua Shi (Changhai Hospital, Second Military Medical University, Shanghai), Yu Gu, Qinfen Su, Yingying Lv (Shanghai Zhabei District Central Hospital), Yun Sun, Qiongpei Gu (Yangpu District Family Planning Service Center), Xixia Pang, Qingwu Zhang (Kong Jiang Hospital of Yangpu District, Shanghai), Songxiao Bai, Baoqiao Qi (Shanghai East City Hospital).

Contributors Substantial contributions to the conception or design of the study were made by GH and WY. YZ and DW prepared the original draft of the manuscript. YZ, DW, YY, YJ, ML, MJ, YD and XC led the study implementation at participating sites. DW and YZ were responsible for the day-to-day project management at each site. XM and WS were responsible for the biobank of the cohort. All authors provided critical reviews of the manuscript for important intellectual content and approved the final version.

Funding This work is supported by the National Key Research and Development Program (grant no. 2016YFC1000506) and Natural Science Foundation of China (grant number 81273168) and Three-year Planning for Strengthening the Construction of Public Health System in Shanghai (GWIV-24).

Competing interests None declared.

Patient consent for publication Not required.

Ethics approval This study was approved by the Ethics Committee of the Children's Hospital of Fudan University, Shanghai, China.

Provenance and peer review Not commissioned; externally peer reviewed.

Data availability statement Data are available upon reasonable request.

Open access This is an open access article distributed in accordance with the Creative Commons Attribution Non Commercial (CC BY-NC 4.0) license, which permits others to distribute, remix, adapt, build upon this work non-commercially, and license their derivative works on different terms, provided the original work is properly cited, appropriate credit is given, any changes made indicated, and the use is non-commercial. See: http://creativecommons.org/licenses/by-nc/4.0/.

ORCID iD

Weili Yan http://orcid.org/0000-0002-7633-7449

\section{REFERENCES}

1 Mahle WT, Newburger JW, Matherne GP, et al. Role of pulse oximetry in examining newborns for congenital heart disease: a scientific statement from the American heart association and American Academy of pediatrics. Circulation 2009;120:447-58.

2 van der Linde D, Konings EEM, Slager MA, et al. Birth prevalence of congenital heart disease worldwide: a systematic review and metaanalysis. J Am Coll Cardiol 2011;58:2241-7.

3 MoPHo C. China's Birth Defects Prevention Report. Beijing, 2012.

4 Zhao Q-ming, Ma X-jing, Ge X-ling, et al. Pulse oximetry with clinical assessment to screen for congenital heart disease in neonates in China: a prospective study. Lancet 2014;384:747-54.

5 Bruneau BG. The developmental genetics of congenital heart disease. Nature 2008;451:943-8.

6 Alverson CJ, Strickland MJ, Gilboa SM, et al. Maternal smoking and congenital heart defects in the Baltimore-Washington infant study. Pediatrics 2011;127:e647-53.

7 Lee LJ, Lupo PJ. Maternal smoking during pregnancy and the risk of congenital heart defects in offspring: a systematic review and metaanalysis. Pediatr Cardiol 2013;34:398-407.

8 Shi M, Wehby GL, Murray JC. Review on genetic variants and maternal smoking in the etiology of oral clefts and other birth defects. Birth Defect Res C 2008;84:16-29.

9 Kučienė R, Dulskienè V. Selected environmental risk factors and congenital heart defects. Medicina 2008;44:827-32.

10 Carmichael SL, Shaw GM. Maternal life event stress and congenital anomalies. Epidemiology 2000;11:30-5.

11 Czeizel AE. Periconceptional folic acid-containing multivitamin supplementation for the prevention of neural tube defects and cardiovascular malformations. Ann Nutr Metab 2011;59:38-40.

12 Botto LD, Mulinare J, Erickson JD. Occurrence of congenital heart defects in relation to maternal multivitamin use. Am J Epidemiol 2000;151:878-84.

13 Ionescu-Ittu R, Marelli AJ, Mackie AS, et al. Prevalence of severe congenital heart disease after folic acid fortification of grain products: time trend analysis in Quebec, Canada. BMJ 2009;338:b1673.

14 Liu S, Joseph KS, Luo W, et al. Effect of folic acid food fortification in Canada on congenital heart disease subtypes. Circulation 2016;134:647-55.

15 Czeizel AE, Dudás I. Prevention of the first occurrence of neural-tube defects by periconceptional vitamin supplementation. N Engl J Med 1992;327:1832-5.

16 Cordero AM, Crider KS, Rogers LM, et al. Optimal serum and red blood cell folate concentrations in women of reproductive age for prevention of neural tube defects: World Health organization guidelines. MMWR Morb Mortal Wkly Rep 2015;64:421-3.

17 Kim Y-I. Will mandatory folic acid fortification prevent or promote cancer? Am J Clin Nutr 2004;80:1123-8.

18 Selhub J, Rosenberg IH. Excessive folic acid intake and relation to adverse health outcome. Biochimie 2016;126:71-8.

19 Wiens D, DeSoto M. Is High Folic Acid Intake a Risk Factor for Autism?-A Review. Brain Sci 2017;7:149.

20 Bolann BJ, Distante S, Mørkrid L, et al. Bloodletting therapy in hemochromatosis: does it affect trace element homeostasis? Journal of Trace Elements in Medicine and Biology 2015;31:225-9.

$21 \mathrm{Hu}$ X-J, Ma X-J, Zhao Q-M, et al. Pulse oximetry and auscultation for congenital heart disease detection. Pediatrics 2017;140:e20171154.

22 Lachin JM. Sample size evaluation for a multiply matched casecontrol study using the score test from a conditional logistic (discrete COX pH) regression model. Stat Med 2008;27:2509-23.

23 Tang YQ. Comments on "Sample size evaluation for a multiply matched case-control study using the score test from a conditional logistic (discrete Cox PH) regression model". Stat Med 2009;28:175-7.

24 Daly LE, Kirke PN, Molloy A, et al. Folate levels and neural tube defects. Implications for prevention. JAMA 1995;274:1698-702.

25 Tam C, McKenna K, Goh YI, et al. Periconceptional folic acid supplementation: a new indication for therapeutic drug monitoring. Ther Drug Monit 2009;31:319-26. 